\title{
Long-term mortality of hospitalized pneumonia in the EPIC- Norfolk cohort
}

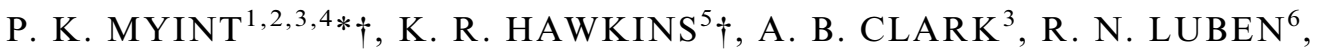 \\ N. J. WAREHAM 7 , K.-T. KHAW ${ }^{4}$ AND A. M. WILSON W $^{3,5}$ \\ ${ }^{1}$ AGEING (Aberdeen Gerontological \& Epidemiological Interdisciplinary Research Group), Epidemiology \\ Group, Institute of Applied Health Sciences, School of Medicine \& Dentistry, University of Aberdeen, Aberdeen, \\ Scotland, UK: ${ }^{2}$ Academic Centre for Applied Clinical \& Translational Research into Ageing (ACTRA), \\ Department of Medicine for the Elderly, Aberdeen Royal Infirmary, NHS Grampian, Aberdeen, Scotland, UK; \\ ${ }^{3}$ Norwich Medical School, University of East Anglia, Norwich Research Park, Norwich, UK, ${ }^{4}$ Clinical \\ Gerontology Unit, Department of Public Health \& Primary Care, School of Clinical Medicine, University of \\ Cambridge, Cambridge, UK; ${ }^{5}$ Department of Respiratory Medicine, Norfolk and Norwich University Hospital, \\ Norwich, UK; ${ }^{6}$ Strangeway Research Laboratory, Department of Public Health \& Primary Care, Cambridge, \\ UK; ${ }^{7}$ MRC-Epidemiology Unit, Institute of Metabolic Science, Addenbrooke's Hospital, Cambridge, UK
}

Received 21 April 2015; Final revision 14 July 2015; Accepted 5 August 2015;

first published online 24 August 2015

\section{SUMMARY}

Little is known about cause-specific long-term mortality beyond 30 days in pneumonia. We aimed to compare the mortality of patients with hospitalized pneumonia compared to age- and sex-matched controls beyond 30 days. Participants were drawn from the European Prospective Investigation into Cancer (EPIC)-Norfolk prospective population study. Hospitalized pneumonia cases were identified from record linkage (ICD-10: J12-J18). For this study we excluded people with hospitalized pneumonia who died within 30 days. Each case identified was matched to four controls and followed up until the end June 2012 (total 15074 person-years, mean $6 \cdot 1$ years, range $0 \cdot 08-15 \cdot 2$ years). Cox regression models were constructed to examine the all-cause, respiratory and cardiovascular mortality using date of pneumonia onset as baseline with binary pneumonia status as exposure. A total of 2465 men and women (503 cases, 1962 controls) [mean age (s.d.) $64.5(8.3)$ years] were included in the study. Between a 30-day to 1-year period, hazard ratios (HRs) of all-cause and cardiovascular mortality were $7 \cdot 3$ [95\% confidence interval (CI) 5.4-9.9] and 5.9 (95\% CI 3.5-9.7), respectively (with very few respiratory deaths within the same period) in cases compared to controls after adjusting for age, sex, asthma, smoking status, pack years, systolic and diastolic blood pressure, diabetes, physical activity, waist-to-hip ratio, prevalent cardiovascular and respiratory diseases. All outcomes assessed also showed increased risk of death in cases compared to controls after 1 year; respiratory cause of death being the most significant during that period (HR 16.4, 95\% CI 8·9-30·1). Hospitalized pneumonia was associated with increased all-cause and specific-cause mortality beyond 30 days.

Key words: All-cause mortality, cardiovascular mortality, long-term mortality, pneumonia, respiratory mortality.

\footnotetext{
* Author for correspondence: Professor P. K. Myint, 4:013 Polwarth Building, School of Medicine \& Dentistry, University of Aberdeen, Foresterhill, Aberdeen AB25 2ZD, UK.

(Email: phyo.myint@abdn.ac.uk)

$\dagger$ These authors served as joint first authors.
}

\section{INTRODUCTION}

Pneumonia is common and in combination with influenza is the fifth leading cause of death in the 
UK [1]. It is the third leading cause of death globally accounting for $7 \cdot 1 \%$ of all deaths along with other lower respiratory tract infections [2]. Pneumonia is also one of the main causes of hospital admissions [3] and according to US statistics up to $10-20 \%$ of all hospitalizations with pneumonia require treatment in the intensive care unit [4]. The short-term mortality from pneumonia is well documented and is consistently between $10 \%$ and $11 \%[5,6]$. Mortality from pneumonia is typically assessed at 30 days or at discharge from hospital [7,8] and patients who have clinical resolution of their symptoms at this time are classified as having survived the episode [9].

It is also suggested that pneumonia produces a chronic inflammatory response which may accelerate the process of cardiovascular disease in the intermediate term [10]. Indeed, it has been shown in a study of 1799 patients hospitalized with pneumonia, that those with raised pro-inflammatory cytokines at discharge had an increased risk of cardiovascular death at 1 year, which was independent of the severity of the pneumonia [10]. More recent studies suggest that pneumonia may influence the patient's longer term survival [11-14]. Mortality was 10\% higher at 1 year for elderly patients with pneumonia compared to other hospitalized survivors [15] and although few studies have analysed specific cause of death postpneumonia diagnosis, cardiovascular disease caused a third of all deaths from 300 patients at 1-year followup in a hospital-based study [10].

It is not known whether pneumonia has impact on longer term mortality in an unselected general population and whether it is linked to cardiovascular cause of death post-1 year follow-up. While there appears to be a greater incidence of cardiovascular disease at 1 year following pneumonia, the risk of cardiovascular disease at different time points post-pneumonia diagnosis has not been assessed. The goals of this study were to examine the association of hospitalized pneumonia on both short ( $\geqslant 30$ days up to 1 year) and long-term allcause and cause-specific mortality focusing on respiratory and cardiovascular causes of death in a general population of middle and older age participants after taking into account potential confounders.

\section{MATERIALS AND METHODS}

\section{Study population}

All study participants were selected from the participants of European Prospective Investigation into
Cancer (EPIC)-Norfolk prospective population-based cohort study. This cohort forms part of a Europe-wide multicentre prospective population study, primarily designed to investigate the relationship between diet and cancer. The study was approved by the Norwich Research Ethics Committee (R\&D reference no. 98CN01).

The methods of the EPIC-Norfolk study have been described in depth elsewhere [16]. The Norfolk population is a mix of urban and rural populations and has high coverage of primary-care physicians (General Practitioners). It is for the most part representative of the UK population; however, there is lower ethnic diversity in this area than the general UK population and the EPIC-Norfolk cohort is $99.6 \%$ white. Briefly, participants were men and women aged 40-79 years recruited between 1993 and 1998. Participants underwent a detailed baseline health examination in which demographic, physiological and behavioural factors were ascertained. This included prevalent illness, smoking status, body mass index (BMI) measured using standard protocols [16]. They were followed up for health events using record linkage.

Hospitalized cases of pneumonia were identified through linkage with hospital records using ICD-10 J12-J18. A total of 1064 cases and 4120 controls (1:4) matched for age, sex and year of recruitment in the EPIC-Norfolk were included. All participants who reported cancer, myocardial infarction and stroke at the EPIC-Norfolk study baseline were excluded. To reduce the bias of excess mortality within 30 days from respiratory cause of death of cases which are identified through death certification from the Office of National Statistics (ONS), we excluded any cases who died within 30 days of hospitalization and their matched controls leaving 503 cases and 1962 controls for the analyses. The start date for analysis for the current study was the date of diagnosis of pneumonia for cases and the same date for case-matched controls. The study follow-up period was from the start date to date of death or end of follow-up (end of June 2012). Prevalent cardiovascular disease (CVD) for the current report was defined as a new diagnosis of CVD after EPIC-Norfolk enrolment but made before the pneumonia date for cases and respective corresponding date for controls.

\section{Outcome measurements}

The primary outcome of interest was all-cause mortality. Secondary outcomes were respiratory mortality and 
cardiovascular mortality. Mortality data were obtained from linkage with ONS in UK. Mortality was assessed between 30 days and $<1$ year for short term and $\geqslant 1$ year for long term [11, 14, 17]. Specific causes of death were coded by a nosologist with ICD-10 codes J00-J99 (ICD-9: 460-496) for respiratory causes, and ICD-10 codes L10-179 (ICD-9: 401-448) for cardiovascular causes, respectively.

\section{Statistical analysis}

Statistical analyses were conducted using Stata v. 11.2/ SE (StatCorp, USA). The baseline characteristics were given as means and standard deviations for normally distributed continuous data and by number and percentages for categorical data. Comparisons between groups at baseline were based on univariable conditional logistic regression models in order to take into account the non-independence due to the matching of cases and controls. Respiratory and cardiovascular mortality in pneumonia cases compared to controls were analysed at different time-frames. The cut-off between short-term and long-term mortality were 1 year and until the end of follow-up, respectively.

The difference in mortality in pneumonia cases compared to controls was examined using Cox regression [18] with a frailty term to account for the non-independence due to the matching of cases and controls. Covariates in the multiple variable models were chosen for the characteristics that differ significantly between group means for at least one cause of mortality using Cox regression on all of the available risk factors (Table 1) by univariate analysis. Multivariate analysis was thus adjusted for history of asthma, smoking status, pack years, forced expiratory volume 1 (FEV1), forced vital capacity (FVC), and physical activity levels (as outlined previously [16]), waist-to-hip ratio, diabetes mellitus, pre-existing CVD and pre-existing respiratory disease. A final model was constructed after additionally adjusting for aspirin use (statin use was not included as there were too few participants who were on statins to provide any meaningful results). No adjustment for age and sex was required due to the matching criteria.

\section{RESULTS}

A total of 503 participants were identified as hospitalized pneumonia cases and these cases were matched by age, gender and year of entry into the EPIC-Norfolk study with 1962 controls (1:3.9) after exclusion of cases who died within 30 days of pneumonia and their respective controls. Participants were followed from the date of diagnosis of pneumonia in cases or the same date for matched controls for a total of 15074 person-years (mean 6.1 years, range $0 \cdot 08-15 \cdot 2$ years). The mean time from entry into EPIC-Norfolk (which began in 1993) and the diagnosis of pneumonia was $9 \cdot 0$ years.

As controls were age- and sex-matched, these baseline characteristics were comparable between cases and controls (Table 1). However, the cases had a significantly higher proportion of current smokers and a higher mean number of pack years than the control group. The prevalence of diabetes mellitus, CVD and respiratory disease, including asthma were significantly higher for cases. Mean FEV1 and FVC were lower within the case group compared to controls. Although the difference in BMI between the two groups was non-significant, the case group had a significantly higher waist-to-hip ratio than controls.

There was a higher incidence of mortality in the case group which was maintained throughout both time-frames and this difference was greatest in terms of respiratory mortality (Table 2). There was an increased risk of mortality for the case group compared to the control group after adjusting for significant risk factors identified (Table 3). There was an increased risk of all-cause mortality and specificcause mortality between 30 days and 1 year, and longer than 1 year. The all-cause mortality rate was highest initially following pneumonia then reduced over time whereas the initial higher respiratory and cardiovascular mortality rates for cases compared to controls was maintained throughout the study period.

The risk of cardiovascular mortality was increased in cases for both time periods, and the highest risk period was between 30 days and 1 year. Interestingly risk of death due to respiratory cause was excessively high 1 year post-pneumonia and the overall risk was driven mainly by this.

Additionally adjusting for aspirin use did not alter the results (data not shown).

\section{DISCUSSION}

Our findings indicate that hospitalized pneumonia is associated with increased risk of mortality not only in the short term $(\geqslant 30$ days to $<1$ year), but also in the longer term ( $>1$ year) compared to age- and sexmatched controls within the general population of 
Table 1. Comparison of characteristics of pneumonia cases and controls without pneumonia

\begin{tabular}{|c|c|c|c|}
\hline Characteristics & Cases & Controls & $P$ value* \\
\hline Total & 503 & 1962 & \\
\hline Males & $269(53 \cdot 5 \%)$ & $1030(52 \cdot 5 \%)$ & n.a. \\
\hline Age, years, (S.D.) & $64 \cdot 7(8 \cdot 4)$ & $64 \cdot 4(8 \cdot 3)$ & n.a. \\
\hline range & $41-77$ & $41-77$ & \\
\hline$<50$ & $35(7 \cdot 0 \%)$ & $132(6 \cdot 7 \%)$ & \\
\hline $50-65$ & $189(37 \cdot 6 \%)$ & $752(38 \cdot 3 \%)$ & \\
\hline$>65$ & $279(55 \cdot 5 \%)$ & $1078(54 \cdot 9 \%)$ & \\
\hline Smoking status & & & $<0 \cdot 001$ \\
\hline Current & $93(18 \cdot 7 \%)$ & $162(8 \cdot 4 \%)$ & $<0 \cdot 001$ \\
\hline Previous & $225(45 \cdot 3 \%)$ & $944(48 \cdot 7 \%)$ & \\
\hline Never & $179(36 \cdot 0 \%)$ & $834(43 \cdot 0)$ & \\
\hline Pack years, mean (s.D.) & $14 \cdot 8(17 \cdot 9)$ & $10 \cdot 4(15 \cdot 2)$ & \\
\hline BMI, mean (S.D.) & $26 \cdot 7(3 \cdot 7)$ & $26 \cdot 64(3 \cdot 7)$ & $0 \cdot 58$ \\
\hline range & $16 \cdot 9-46 \cdot 4$ & $16 \cdot 0-43 \cdot 5$ & $0 \cdot 12$ \\
\hline$<18 \cdot 5$ & $5(1 \cdot 0)$ & $9(0 \cdot 5)$ & \\
\hline$\geqslant 18 \cdot 5-25$ & $174(34 \cdot 6)$ & $681(34 \cdot 7)$ & \\
\hline$>25-30$ & $226(44 \cdot 9)$ & $949(48 \cdot 4)$ & \\
\hline$>30$ & $98(19 \cdot 5)$ & $321(16 \cdot 4)$ & \\
\hline Asthma & $73(14 \cdot 5)$ & $151(7 \cdot 7)$ & $<0.001$ \\
\hline Diabetes mellitus & $26(5 \cdot 2)$ & $52(2 \cdot 7)$ & $0 \cdot 005$ \\
\hline $\begin{array}{l}\text { FEV1, mean (s.D.) } \\
\qquad(N=5037)\end{array}$ & $210 \cdot 51(75 \cdot 0)$ & $235 \cdot 66(70 \cdot 93)$ & $<0 \cdot 001$ \\
\hline $\begin{array}{l}\text { FVC, mean (S.D.) } \\
\quad(N=5037)\end{array}$ & $268 \cdot 4(93.93)$ & $291 \cdot 2(90 \cdot 6)$ & $<0.001$ \\
\hline $\begin{array}{l}\text { Systolic, mean (s.D.) } \\
\qquad(N=5170)\end{array}$ & $141 \cdot 1(19 \cdot 5)$ & $140 \cdot 5(18 \cdot 9)$ & $0 \cdot 60$ \\
\hline Alcohol intake, mean (s.D.) & & & $0 \cdot 30$ \\
\hline$(N=5017)$ & $8 \cdot 5(13 \cdot 0)$ & $7 \cdot 9(11 \cdot 7)$ & \\
\hline Inactive & $351(69 \cdot 8)$ & $1243(63 \cdot 4)$ & $0 \cdot 004$ \\
\hline Active & $152(30 \cdot 2)$ & $719(36 \cdot 7)$ & \\
\hline Social class & & & $0 \cdot 162$ \\
\hline I & $26(5 \cdot 3)$ & $132(7 \cdot 0)$ & \\
\hline II-IV & $440(90 \cdot 2)$ & $1703(89 \cdot 9)$ & \\
\hline $\mathrm{V}$ & $22(4 \cdot 5)$ & $59(3 \cdot 1)$ & \\
\hline Cholesterol, mean (S.D.) & $6 \cdot 2(1 \cdot 2)$ & $6 \cdot 3(1 \cdot 2)$ & $0 \cdot 19$ \\
\hline Waist:hip ratio, mean (s.D.) & $0.88(0.09)$ & $0 \cdot 87(0 \cdot 09)$ & $0 \cdot 001$ \\
\hline Statin use & $6(1 \cdot 2)$ & $15(0 \cdot 8)$ & $0 \cdot 076$ \\
\hline Aspirin use & $43(8 \cdot 6)$ & $124(6 \cdot 3)$ & $0 \cdot 319$ \\
\hline $\begin{array}{l}\text { Respiratory disease prior to censored } \\
\text { pneumonia }\end{array}$ & $73(14 \cdot 5)$ & $300(15 \cdot 3)$ & \\
\hline $\begin{array}{l}\text { Respiratory disease within } 1 \text { year prior to } \\
\text { pneumonia date }\end{array}$ & $46(9 \cdot 2)$ & $36(1 \cdot 8)$ & \\
\hline $\begin{array}{l}\text { Cardiovascular disease prior to } \\
\text { pneumonia date } \dagger\end{array}$ & $168(33 \cdot 4)$ & $449(22 \cdot 9)$ & \\
\hline $\begin{array}{l}\text { Cardiovascular disease within } 1 \text { year } \\
\text { prior to censored pneumonia date }\end{array}$ & $57(11 \cdot 3)$ & $72(3 \cdot 7)$ & \\
\hline
\end{tabular}

n.a., Not applicable.

Data presented are mean (S.D.) for continuous data and number (\%) for categorical data.

* Indicates that overall $P$ values are provided for categorical comparisons where there are more than two categories

$\dagger$ Prevalent myocardial infarction and stroke at the time of baseline of the EPIC-Norfolk study were excluded from the outset and these numbers indicate the number (\%) of participants with incident cardiovascular disease diagnosed between EPIC-Norfolk study enrolment and pneumonia date (baseline of the current report). 
Table 2. Short, intermediate and long-term crude mortality rates for both pneumonia cases and controls by cause of death

\begin{tabular}{|c|c|c|c|c|c|c|c|}
\hline \multirow[b]{3}{*}{ Time and cause of mortality } & \multicolumn{3}{|l|}{ Cases } & \multicolumn{3}{|l|}{ Controls } & \multirow[b]{3}{*}{$P$ value } \\
\hline & \multirow[b]{2}{*}{$N$ at risk } & \multicolumn{2}{|c|}{ Deaths } & \multirow[b]{2}{*}{$N$ at risk } & \multicolumn{2}{|c|}{ Deaths } & \\
\hline & & $N$ & $(\%)$ & & $N$ & $(\%)$ & \\
\hline \multicolumn{8}{|l|}{ All-cause mortality } \\
\hline Total & 503 & 304 & $(60 \cdot 4)$ & 1962 & 488 & $(24 \cdot 9)$ & $<0.001$ \\
\hline$\geqslant 30$ days to $<1$ year & 503 & 122 & $(24 \cdot 3)$ & 1962 & 72 & $(3 \cdot 7)$ & $<0 \cdot 001$ \\
\hline$\geqslant 1$ year & 381 & 182 & $(47 \cdot 8)$ & 1890 & 416 & $(22 \cdot 0)$ & $<0.001$ \\
\hline \multicolumn{8}{|l|}{ Respiratory mortality } \\
\hline Total & 503 & 63 & $(12 \cdot 5)$ & 1962 & 21 & $(1 \cdot 1)$ & $<0.001$ \\
\hline$\geqslant 30$ days to $<1$ year & 503 & 19 & $(3 \cdot 8)$ & 1962 & 4 & $(0 \cdot 2)$ & $<0 \cdot 001$ \\
\hline$\geqslant 1$ year & 381 & 44 & $(11 \cdot 5)$ & 1890 & 17 & $(0 \cdot 9)$ & $<0.001$ \\
\hline \multicolumn{8}{|l|}{ Cardiovascular mortality } \\
\hline Total & 503 & 83 & $(16 \cdot 5)$ & 1962 & 177 & $(9 \cdot 0)$ & $<0.001$ \\
\hline$\geqslant 30$ days to $<1$ year & 503 & 39 & $(7 \cdot 8)$ & 1962 & 28 & $(1 \cdot 4)$ & $<0 \cdot 001$ \\
\hline$\geqslant 1$ year & 381 & 44 & $(11 \cdot 5)$ & 1890 & 149 & $(7.9)$ & $0 \cdot 013$ \\
\hline
\end{tabular}

Table 3. Hazard ratios and corresponding 95\% confidence intervals of incident mortality for cases using the control group as a reference category

\begin{tabular}{|c|c|c|c|c|c|c|c|c|c|}
\hline \multirow[b]{2}{*}{ Time } & \multicolumn{3}{|c|}{ All-cause mortality } & \multicolumn{3}{|c|}{ Respiratory mortality } & \multicolumn{3}{|c|}{ Cardiovascular mortality } \\
\hline & HR & $(95 \% \mathrm{CI})$ & $P$ value & HR & $(95 \% \mathrm{CI})$ & $P$ value & HR & $(95 \% \mathrm{CI})$ & $P$ value \\
\hline Total & $4 \cdot 0$ & $(3 \cdot 4-4 \cdot 7)$ & $<0 \cdot 001$ & $20 \cdot 4$ & $(11 \cdot 7-35 \cdot 8)$ & $<0 \cdot 001$ & $2 \cdot 6$ & $(2 \cdot 0-3 \cdot 5)$ & $<0 \cdot 001$ \\
\hline$\geqslant 30$ days to $<1$ year & $7 \cdot 3$ & $(5 \cdot 4-9 \cdot 9)$ & $<0 \cdot 001$ & & & & $5 \cdot 9$ & $(3 \cdot 5-9 \cdot 7)$ & $<0 \cdot 001$ \\
\hline$\geqslant 1$ year & $2 \cdot 8$ & $(2 \cdot 3-3 \cdot 4)$ & $<0 \cdot 001$ & $16 \cdot 4$ & $(8 \cdot 9-30 \cdot 1)$ & $<0 \cdot 001$ & $1 \cdot 6$ & $(1 \cdot 1-2 \cdot 3)$ & 0.02 \\
\hline
\end{tabular}

HR, Hazard ratio; CI, confidence interval.

Covariates adjusted are smoking status, pack years, physical activity levels, waist to hip ratio, diabetes mellitus, prevalent cardiovascular disease and prevalent respiratory disease for all modes of mortality and all time frames. Matching is account for by a shared frailty term of each matched set.

middle and older age. This increase in mortality persisted throughout the entire length of follow-up for allcause respiratory, and cardiovascular mortality. Risk of death due to cardiovascular cause appeared to be particularly high within 1 year of pneumonia and increased risk of mortality from respiratory causes appeared to be very high after 1 year in cases (who did not die within 1 month) compared to the control population who did not have hospitalized pneumonia during the study period.

Our findings support previous studies. Recently, it was reported that hospitalization for pneumonia was associated with increased short-term and long-term risk of CVD, suggesting that pneumonia may be a risk factor for CVD [19]. Furthermore, Kaplan et al., found that mortality in elderly patients hospitalized with community-acquired pneumonia (CAP) was 10\% higher at 1 year than those hospitalized for other reasons [15]. Bordon et al., based on their study on a hospitalized population, also found an increased 7-year all-cause mortality in patients hospitalized with CAP compared to hospitalized controls [20].

A cohort study, also using the general population as controls, found that the patients with pneumonia were almost 46 times as likely to die in the first 30 days as participants without pneumonia [17]. They found that for pneumonia cases the adjusted risk of all-cause mortality over total follow-up period was $4 \cdot 6$, which is in line with our findings.

This study further supports the evidence that the increase in cardiovascular risk persists past 1 year. In light of this finding, patients admitted to hospital 
with pneumonia should have a cardiac risk assessment including medication review, to consider interventions for CVD prevention if appropriate. The reason why people with pneumonia had increased cardiovascular mortality risk is unclear. However, there are plausible mechanisms which could lead to this observed link between pneumonia and subsequent cardiovascular mortality. Pneumonia increases the levels of inflammatory cytokines promoting thrombogenesis [21] and reduces ventricular function [22, 23], both of which could lead to an increase in cardiac events in the short term. It is also thought that pneumonia produces a chronic inflammatory response which would accelerate the process of CVD in the intermediate term [10]. It was shown that patients hospitalized with pneumonia who experience persistently raised pro-inflammatory cytokines at discharge had an increased risk of cardiovascular death at 1 year, independent of the severity of illness [10].

This study has some limitations. First, neither the clinical severity of pneumonia nor the virulence of the disease-causing organisms was accounted for. Neither were the different levels of inflammatory markers which indicate an extent of host immune response, both of which have had a demonstrated effect on mortality [10, 14]. A possible weakness is the misclassification of pneumonia diagnosis due to variable coding practices and lack of confirmatory radiological evidence as a requirement for inclusion. Evidence is unclear as to how accurate the pneumonia diagnosis is in hospital [24]. However, the main misclassified diagnosis is likely to be other causes of lower respiratory tract infection (LRTI) and this inclusion bias would result in a lower mortality within the case group as lobar pneumonia is the LRTI with the highest mortality rate [25], thus the observed associations if any would be attenuation of the actual impact of pneumonia. We identified only individuals with hospitalized pneumonia so those with episodes of pneumonia treated only in the community were not included. Hospitalized pneumonia cases are likely to be more severe so the generalizability of findings to all pneumonias occurring in the community is limited. To reduce such bias associated with high mortality due to pneumonia-related death (i.e. respiratory cause) we deliberately excluded cases within EPIC-Norfolk who died within 30 days of pneumonia diagnosis. We were also unable to examine the specific cause of CVD mortality.

People who are admitted to hospital for pneumonia are also more likely to have comorbidities which may predispose to increased mortality risk. Although we adjusted for many covariates including lung function as well as history of asthma and chronic respiratory illnesses, there may be unknown confounders or there may be residual confounding effect of known confounders. While any hospitalization may increase the risk of mortality, a more relevant issue perhaps is whether people who had pneumonia were associated with increased cardiovascular risk in the longer term. We acknowledge that we were not able to examine this issue in people with pneumonia who were not hospitalized. Indeed, it is possible that a proportion of controls might have had pneumonia which did not result in hospitalization during follow-up of the study. This misclassification as well as any hospitalization with increased risk of death, however, would only attenuate the results. Finally, in clinical practice especially in older people conditions such as pneumonia and heart failure co-exist and there may be possibility of mis-diagnosis. Nevertheless, such error is likely to contribute mostly to $\leqslant 30$-day mortality and is unlikely to influence the $>30$-day relationships between pneumonia and mortality outcome.

The EPIC-Norfolk cohort comprised communitydwelling middle- and older-aged participants who agreed to participate at baseline recruitment, therefore there may be healthy responder bias. However, truncation of sample distribution would be likely to attenuate the findings.

The participants' baseline characteristics (e.g. FEV1/ FEV) were taken from entry into the EPIC-Norfolk cohort which predated the current study entry date by a mean of $9 \cdot 0$ years. There could be significant differences between characteristics at entry into the EPIC-Norfolk study and at the point of pneumonia diagnosis, resulting in measurement error in the covariables. However we were able to control for wide-ranging demographic, lifestyle and medical factors including prevalent cardiovascular and chronic lower respiratory tract illnesses.

In summary, hospitalized pneumonia is associated with subsequent increased risk of mortality and this persists beyond 1 year after diagnosis in middle and older ages. Clinicians should be aware of the increased mortality from not only respiratory causes but also cardiovascular causes.

\section{ACKNOWLEDGEMENTS}

The EPIC-Norfolk study is supported by programme grants from the Medical Research Council (G1000143) and Cancer Research UK (8257). The funders had no 
role in study design, analysis, and interpretation of the findings.

We gratefully acknowledge the participants of the study and participating General Practitioners. We also thank our funders.

\section{DECLARATION OF INTEREST}

None.

\section{REFERENCES}

1. Office for National Statistics. Deaths registered in England and Wales in 2010, by cause. 2011 (http:// www.ons.gov.uk/ons/rel/vsob1/mortality-statistics--deat hs-registered-in-england-and-wales--series-dr-/2010/stbdeaths-by-cause-2010.html\#tab-Leading-causes-of-mor tality-in-2010). Accessed 22 February 2013.

2. Mathers CD, Fat DM, Boerma JT, The global burden of disease: 2004 update. World Health Organization, 2008.

3. Guest JF, Morris A. Community-acquired pneumonia: the annual cost to the National Health Service in the UK. European Respiratory Journal 1997; 10: 1530-1534.

4. File Jr. TM, Marrie TJ. Burden of community-acquired pneumonia in North American adults. Postgraduate Medicine 2010; 122: 130-141.

5. Lave JR, et al. Hospitalized pneumonia. Outcomes, treatment patterns, and costs in urban and rural areas. Journal of General Internal Medicine 1996; 11: 415-421.

6. Marrie TJ, et al. Mortality during hospitalisation for pneumonia in Alberta, Canada, is associated with physician volume. European Respiratory Journal 2003; 22: 148-55.

7. Eurich DT, Majumdar SR, Marrie TJ. Population-based cohort study of outpatients with pneumonia: rationale, design and baseline characteristics. BMC Infectious Diseases 2012; 12: 135.

8. Hoo Lee J, Hyung Kim Y. Comparison of clinical characteristics between healthcare-associated pneumonia and community-acquired pneumonia in patients admitted to secondary hospitals. Brazilian Journal of Infectious Diseases 2012; 16: 321-328.

9. Ramirez JA, Anzueto AR. Changing needs of community-acquired pneumonia. Journal of Antimicrobial Chemotherapy 2011; 66 (Suppl. 3): 3-9.

10. Yende $\mathbf{S}$, et al. Inflammatory markers at hospital discharge predict subsequent mortality after pneumonia and sepsis. American Journal of Respiratory and Critical Care Medicine 2008; 177: 1242-1247.
11. Yende $\mathbf{S}$, et al. Influence of comorbid conditions on long-term mortality after pneumonia in older people. Journal of the American Geriatrics Society 2007; 55: 518-525.

12. Mortensen EM, et al. Assessment of mortality after longterm follow-up of patients with community-acquired pneumonia. Clinical Infectious Diseases 2003; 37: 1617-24.

13. Waterer GW, Kessler LA, Wunderink RG. Medium-term survival after hospitalization with community-acquired pneumonia. American Journal of Respiratory and Critical Care Medicine 2004; 169: 910-914.

14. Johnstone $\mathbf{J}$, et al. Long-term morbidity and mortality after hospitalization with community-acquired pneumonia: a population-based cohort study. Medicine (Baltimore) 2008; 87: 329-334.

15. Kaplan V, et al. Pneumonia: still the old man's friend? Archives of Internal Medicine 2003; 163: 317-23.

16. Day N, et al. EPIC-Norfolk: study design and characteristics of the cohort. European Prospective Investigation of Cancer. British Journal of Cancer 1999; 80 (Suppl. 1): 95-103.

17. Myles PR, et al. Pneumonia mortality in a UK general practice population cohort. European Journal of Public Health 2009; 19: 521-526.

18. Cox D. Regression models and life tables. Journal of the Royal Statistical Society 1972; 34: 187-220.

19. Corrales-Medina VF, et al. Association between hospitalization for pneumonia and subsequent risk of cardiovascular disease. Journal of the American Medical Association 2015; 313: 264-274.

20. Bordon J, et al. Decrease in long-term survival for hospitalized patients with community-acquired pneumonia. Chest 2010; 138: 279-283.

21. Mendall MA, et al. Relation of serum cytokine concentrations to cardiovascular risk factors and coronary heart disease. Heart 1997; 78: 273-277.

22. Kumar A, et al. Tumor necrosis factor alpha and interleukin-1beta are responsible for in vitro myocardial cell depression induced by human septic shock serum. Journal of Experimental Medicine 1996; 183: 949-958.

23. Mann DL. Inflammatory mediators and the failing heart past, present, and the foreseeable future. Circulation Research 2002; 91: 988-998.

24. Pink KL, Mitchell I, Davies HE. P17 the accuracy of a diagnosis of pneumonia in a UK teaching hospital. Thorax 2012; 67 (Suppl. 2): A71-A71.

25. Moellering RC. The continuing challenge of lower respiratory tract infections. Clinical Infectious Diseases 2002; 34 (Suppl. 1): S1-S3. 